\title{
Neurologists' attitudes and options for anticoagulation therapy in central China
}

\author{
Jing Shen ${ }^{1}$, Man $\mathrm{Li}^{1}$, Shiyi $\mathrm{Cao}^{2}$, Zuxun $\mathrm{Lu}^{2}$, Yuanpeng Xia ${ }^{1}$, Shengcai Chen ${ }^{1}$, Ying $\mathrm{Bi}^{1}$, \\ Zhuoyuan $\mathrm{Cai}^{1}$, Bo $\mathrm{Hu}^{1}$, and Fei $\mathrm{Cao}^{1}$ \\ ${ }^{1}$ Wuhan Union Hospital \\ ${ }^{2}$ Huazhong University of Science and Technology Tongji Medical College School of Public \\ Health
}

July 16, 2020

\begin{abstract}
Aims: We aim to find out the factors affecting the use of anticoagulants and the intensity of their choices, and to establish a basis for improving neurologists' effective implementation of the guidelines. Methods: A cross-sectional study is conducted in Hubei province in central China. Each neurologist completes a standard-structured anonymous questionnaire through face-to-face interviews. The problems include the attitude and options about anticoagulant therapy. Results: A total of 611 neurologists from 38 hospitals respond to this survey. For the best treatment of atrial fibrillation, more than $80 \%$ of physicians choose anticoagulant therapy. For patients with atrial fibrillation and cerebral infarction, physicians think Warfarin is the preferred drug as high as $93.8 \%$. Among the anticoagulant drugs ever used by clinicians, the use rate of Warfarin is $93.8 \%$, but the use rate of direct oral anticoagulants is extremely low. The use of direct oral anticoagulants is related to the educational level and the geographical location of the hospital. Bleeding risk is the first reason influencing clinicians' choice of Warfarin, accounts for 88.9\%. $97.7 \%$ of the clinicians recommend patients with Warfarin regularly monitoring the INR, but the frequency of monitoring is inconsistent. Clinicians have a high willingness to learn about AF, but the proportion of hospitals carry out appropriate training is low. Conclusions: There are still some gaps with the guidelines on the choice of anticoagulant drugs. Neurologists have positive attitude towards anticoagulant therapy and a strong willingness to learn, but the corresponding training is lacking. Continuous professional training is necessary.
\end{abstract}

\section{Neurologists' attitudes and options for anticoagulation therapy in central China}

Jing Shen ${ }^{1,3}, \mathrm{MD}, \mathrm{PhD}, \mathrm{Man} \mathrm{Li}^{1}, \mathrm{MD}, \mathrm{PhD}$, Shiyi $\mathrm{Cao}^{2}, \mathrm{MD}$, Zuxun $\mathrm{Lu}^{2}, \mathrm{MD}$, Yuanpeng Xia ${ }^{1}, \mathrm{MD}, \mathrm{PhD}$, $\mathrm{PhD}$, Shengcai Chen ${ }^{1}, \mathrm{MD}, \mathrm{PhD}$, Ying Bi ${ }^{1}, \mathrm{MD}$, Zhuoyuan $\mathrm{Cai}^{1}$, MD, Bo $\mathrm{Hu}^{1,{ }^{*}}, \mathrm{MD}, \mathrm{PhD}$, Fei Cao ${ }^{1, *}, \mathrm{MD}$, $\mathrm{PhD}$

${ }^{1}$ Department of Neurology, Union Hospital, Tongji Medical College, Huazhong University of Science and Technology, Wuhan, China

${ }^{2}$ School of Public Health, Tongji Medical College, Huazhong University of Science and Technology, Wuhan, China

${ }^{3}$ Department of Neurology, The First Affiliated Hospital, School of Medicine, Shihezi University, Shihezi, China

Jing Shen and Man Li should be considered joint first author.

$\mathrm{Bo} \mathrm{Hu}$ and Fei Cao should be considered joint senior author.

*Corresponding author 
Short title:Neurologists' options for anticoagulant

\section{Correspondence:}

Fei Cao, MD, PhD

Department of Neurology, Union Hospital, Tongji Medical College, Huazhong University of Science and Technology, Wuhan, 1277 Jiefang Avenue, Wuhan 430022, China

Email:2000xh0644@hust.edu.cn

Tel: $01186-27-85726028$

Fax: 01186-27-85726028

$\mathrm{Bo} \mathrm{Hu}, \mathrm{MD}, \mathrm{PhD}$

Department of Neurology, Union Hospital, Tongji Medical College, Huazhong University of Science and Technology, Wuhan, 1277 Jiefang Avenue, Wuhan 430022, China

Email:hubo@mail.hust.edu.cn

Tel: $01186-27-85726028$

Fax: 01186-27-85726028

\section{Acknowledgments}

We appreciate following units to participating this study: Jingmen No.1 people's hospital, Affiliated Taihe hospital of Hubei University of Medicine, The Central Hospital of Wuhan, Xiangyang No.1 people's hospital, The first people's hospital of Jingzhou, Wuhan No.1 Hospital, Hubei provincial people's hospital, Xiangyang Central Hospital, Jingzhou central hospital, Wuhan General Hospital of the Guangzhou Military Region of the Chinese People's Liberation Army, The Central Hospital of EnShi Tujia and Miao autonomous Prefecture, Wuhan Pu'ai Hospital East Campus, Wuhan Pu'ai Hospital, Suizhou Central Hospital, Zaoyang No.1 people's hospital, Dongfeng Motor Corporation General Hospital, Central Hospital of Yangxin County, General hospital of the Yangtze River Shipping, Ezhou Central Hospital, Central Hospital of Hefeng County, Jingmen No.2 people's hospital, The ninth hospital of Wuhan, The sixth hospital of Wuhan, The people's hospital of Honghu, Yichang Huimin Hospital, Enshi Central Hospital, Hanchuan people's hospital, Huanggang Central Hospital, Songzi people's hospital.(Ranking order is based on the number of cases provided)

\section{Funding}

This research was supported in part by the National Key Research and Development Program of China (no. 2018YFC1312200) and National Natural Science Foundation of China (no. 81820108010 and no. 81571110 to BH, no. 81771249 to YPX). Youth Program of National Natural Science Foundation of China (no. 81801172 for SCC). National Research Foundation for the Doctoral Program of Higher Education of China (no. 20120142110068 to BH), and New Century Excellent Talents in University (no. NCET-10-0406 to BH).

\section{Disclosures}

All authors declare that they have no conflict of interest.

\section{Author contribution}

Bo H, Fei C, concept/design, and final approval. Jing S,Man L, data analysis/interpretation, drafting article, Shiyi C, Zuxun L, critical revision of article, critical review, Yuanpeng X, Shengcai C, data analysis/interpretation. Ying B, Zhuoyuan C, data collection and analysis;

\section{Hosted file}

main text for IJCP.docx available at https://authorea.com/users/343368/articles/470041neurologists-attitudes-and-options-for-anticoagulation-therapy-in-central-china 
Fig.1A
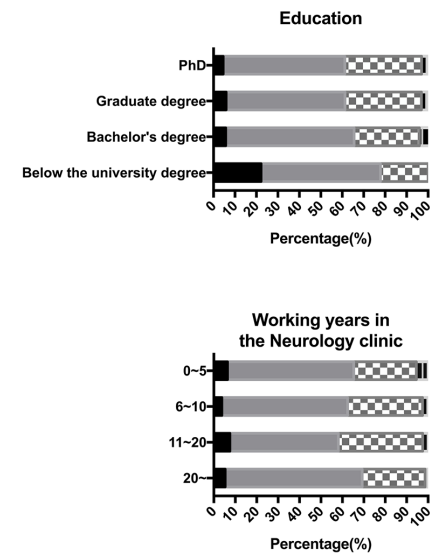

Fig.1B

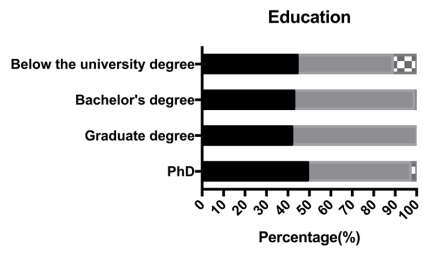

Working years in
the Neurology clinic

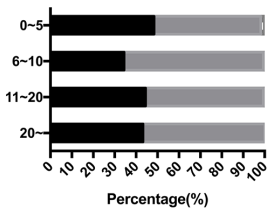

II. Once every three months

Once every month

Once every 2 weeks

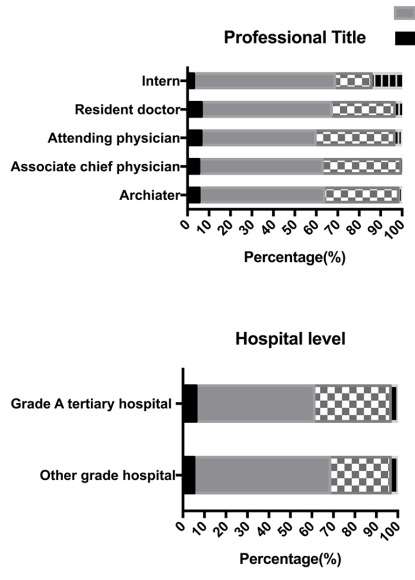

Hospital without monitoring equipment

En Poor patient compliance

Professional Title Trouble in the monitoring process

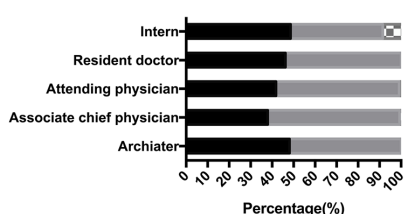

Percentage(\%)

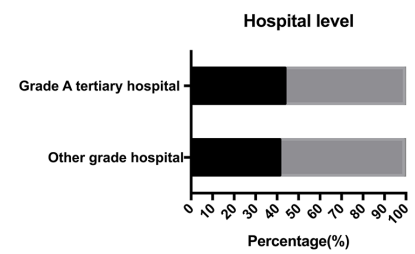

